\title{
Shock compression of quartz in the high-pressure fluid regime
}

D. G. Hicks, T. R. Boehly, P. M. Celliers, J. H. Eggert, E. Vianello, D. D. Meyerhofer, G. W. Collins

December 11, 2006

Physics of Plasmas 
This document was prepared as an account of work sponsored by an agency of the United States Government. Neither the United States Government nor the University of California nor any of their employees, makes any warranty, express or implied, or assumes any legal liability or responsibility for the accuracy, completeness, or usefulness of any information, apparatus, product, or process disclosed, or represents that its use would not infringe privately owned rights. Reference herein to any specific commercial product, process, or service by trade name, trademark, manufacturer, or otherwise, does not necessarily constitute or imply its endorsement, recommendation, or favoring by the United States Government or the University of California. The views and opinions of authors expressed herein do not necessarily state or reflect those of the United States Government or the University of California, and shall not be used for advertising or product endorsement purposes. 


\title{
Shock compression of quartz in the high-pressure fluid regime
}

\author{
D. G. Hicks,,${ }^{1, *}$ T. R. Boehly, ${ }^{2}$ P. M. Celliers,${ }^{1}$ J. H. Eggert,${ }^{1}$ \\ E. Vianello, ${ }^{2, \dagger}$ D. D. Meyerhofer, ${ }^{2, \ddagger}$ and G. W. Collins ${ }^{1}$ \\ ${ }^{1}$ Lawrence Livermore National Laboratory, Livermore, CA 94550 \\ ${ }^{2}$ Laboratory for Laser Energetics, University of Rochester, NY 14623
}

\begin{abstract}
The Hugoniot of quartz has been measured using laser-driven shock waves with pressures from 2 to 15 Mbar. Within this pressure range silica transforms from a liquid near melt into a dense plasma. Results are in good agreement with previous studies in part of this range performed using explosive- and nuclear-driven shocks indicating the absence of time-dependent effects for timescales between several hundred picoseconds and several hundred microseconds. These data combined with earlier data at lower pressures clearly show the increasing compressibility of silica as it transitions from solid to liquid to dense plasma regimes.
\end{abstract}

\footnotetext{
*Author to whom correspondence should be addressed: hicks13@llnl.gov ${ }^{\dagger}$ Also Department of Physics and Astronomy, University of Rochester.

¥Also Department of Physics and Astronomy and Department of Mechanical Engineering, University of Rochester.
} 


\section{INTRODUCTION}

Silica presents a prototypical system for studying high pressure structural changes, one that is of fundamental importance to the study of the Earth's interior [1]. Its phase diagram, revealed by a wide array of static [2-6] and dynamic [7-10] compression experiments, shows a transformation from tetrahedral to octahedral coordination with increasing pressure, although many details remain controversial [11-13]. The availability of several silica polymorphs at ambient pressure - in particular fused silica, quartz, cristobalite, coesite, and stishovite - in addition to porous samples has made dynamic compression experiments especially valuable since the different ambient densities allow shock Hugoniots to traverse a wide range of phase space $[12,14]$. While extensive shock data exist for the various solid phases of silica, only limited data $[15,16]$ exist for the Hugoniot of the high-pressure fluid. Shock melting of $\alpha$-quartz, detected using temperature measurements, was observed at 1.1 Mbar [9]; above this pressure silica is expected to transform continuously from a liquid into a dense plasma, a process that has recently been investigated in molecular dynamics simulations [17]. Precise measurement of the Hugoniot through this dissociation regime is fundamental to the modelling of large meteor impacts [18] and provides a bound on the behavior of liquid silicates that likely exist at the earth's core-mantle boundary [19, 20]. Such studies are critical to developing an understanding of how covalently-bonded, condensed matter systems evolve into dense, strongly-coupled plasmas at high pressure and temperature.

In this study we perform extensive measurements of the $\alpha$-quartz Hugoniot from 2 to 15 Mbar using laser-driven shock waves. A fundamental question that arises in dynamic compression experiments is whether the states probed are at equilibrium. This issue is of particular relevance to laser-driven shocks where ultra-high pressures are achieved in less than a nanosecond. In comparison [21], nuclear-driven shock experiments were performed over timescales of tens of microseconds [10], laboratory explosive and gas gun studies over fractions of microseconds [9, 10], and magnetically-driven flyer plates over tens of nanoseconds [22]. Since there exist both explosive- and nuclear-driven data on $\alpha$-quartz in the range 2 to 6 Mbar, and also a single nuclear experiment at 20 Mbar [15, 16], this material presents a valuable medium for examining the validity of the equilibrium assumption in laser-driven equation-of-state (EOS) studies. Although shocked quartz has been shown to exhibit non-equilibrium behavior in its solid phases $[9,23]$ we find that in the fluid regime of 
silica excellent agreement exists amongst these different experiments whose timescales differ by more than 4 orders of magnitude. We believe this to be the most precise comparison of Hugoniot measurements on different shock wave platforms achieved to date.

\section{METHODS}

The Hugoniot measurements in this experiment use the impedance-match technique [24] with aluminum as the material standard. Determining the pressure and density in the sample requires knowledge of the Hugoniot and release behavior of aluminum. This and a similar inverse approach were used in previous experiments $[15,16]$ to which our data will be compared. In other respects our experiments differ significantly from the earlier ones: in both time and length scales, in the drive mechanism, and in the detector systems.

This experiment was performed on the OMEGA laser at the University of Rochester, a neodymium-doped phosphate glass system that operates with frequency-tripled, $0.35 \mu \mathrm{m}$ light [25]. To generate the shock pressures explored in these experiments, laser energies of 440 to $3100 \mathrm{~J}$ were delivered using $3.7 \mathrm{~ns}$ square pulse. The laser focal region had a super-gaussian distribution with a $600 \mu \mathrm{m}$ diameter central region of uniform intensity. Targets consisted of a z-cut, $\alpha$-quartz sample mounted on the lower step of a diamondturned aluminum pusher (see Fig. 1a). A plastic ablator was used to minimize hard x-ray generation in the laser-plasma region. Three different thicknesses were used for the ablatorpusher combination: $20 \mu \mathrm{m}$ of $\mathrm{CH}$ on a $90-130 \mathrm{Al}$ step (90 $\mu \mathrm{m}$ lower step and $130 \mu \mathrm{m}$ upper step); $20 \mu \mathrm{m}$ of $\mathrm{CH}$ on a $50-85 \mu \mathrm{m} \mathrm{Al} \mathrm{step,} \mathrm{and} 20 \mu \mathrm{m} \mathrm{CH}$ plus $80 \mu \mathrm{m}$ of $\mathrm{CH}-\mathrm{Br}$ (plastic with $2 \% \mathrm{Br}$ by atomic weight) on a $50-85 \mu \mathrm{m} \mathrm{Al}$ step. The density of quartz was measured to be $2.65 \mathrm{~g} / \mathrm{cm}^{3}$ and the refractive index along its c-axis at $532 \mathrm{~nm}$ was found to be 1.547 . The height of the step in the aluminum pusher was measured in each target using a white-light interferometer.

The shock diagnostic was a line-imaging Velocity Interferometer System for Any Reflector (VISAR) [26, 27] which measures the Doppler shift of a moving reflector. Implementation of this system on the OMEGA laser is described in detail in reference [28]. Two VISAR's with different velocity sensitivities were used to resolve $2 \pi$ phase shift ambiguities that occur at shock break-out from the aluminum. The velocity sensitivities for the two VISAR instruments, which used $7.2 \mathrm{~mm}$ and $3.1 \mathrm{~mm}$ thick fused silica etalons, was 4.465 and 10.400 
$\mu \mathrm{m} / \mathrm{ns} /$ fringe in quartz. Post-processing of the VISAR images can determine the fringe position to $\sim 5 \%$ of a fringe; since the measured shock velocities are $14-24 \mu \mathrm{m} / \mathrm{ns}$ in quartz, the multiple fringe shifts allow the precision of the shock velocity measurement to be $\sim 1-2 \%$. The probe source was an injection-seeded, Q-switched, yttrium-aluminum garnet laser, operating at a wavelength of $532 \mathrm{~nm}$ with a pulse length of $\sim 25 \mathrm{~ns}$. Streak cameras with temporal windows of $\sim 3$ ns were used to detect the reflected probe signal. The time resolution of the VISAR and streak camera system was 40-50 ps.

\section{RESULTS}

A sample VISAR trace is shown in Fig. 1b. This single time-resolved image contains the two experimental observables used to determine an impedance-matched EOS point in quartz: aluminum, $U_{s}^{\mathrm{Al}}$, and quartz, $U_{s}^{\mathrm{Q}}$, shock speeds. The average $U_{s}^{\mathrm{Al}}$ is determined by the transit time across the aluminum step; the time-resolved $U_{s}^{\mathrm{Q}}$ is determined by the fringe shifts once the shock wave enters the quartz, caused by a Doppler shift at the reflecting shock front. Pressures were high enough on all shots that the shock front in quartz had reflectivities greater than a few percent [29]. The resulting shock velocity history in quartz is shown in Fig. 1.

Errors in $U_{s}^{\mathrm{Q}}$ are 1-2 \% and are dominated by the uncertainty in determining a fringe shift as described above. Errors in $U_{s}^{\mathrm{Al}}$ are $\sim 1.5-2.5 \%$ and give rise to the largest uncertainty in the shock density inferred by impedance-matching. The primary contribution to this error comes from measuring the aluminum break-out time at each step. Typically this uncertainty is about $15-25$ ps which, for the streak camera sweep speeds used in this experiment, corresponds to 5-8 pixels on the VISAR record [30]. For transit times ranging from $1.2-2.5 \mathrm{~ns}$ this gives an uncertainty of 1-2 \%. Other components of the transit time uncertainty include the step height measurement $( \pm 0.2 \mu \mathrm{m}$, giving $\sim 0.6 \%$ error $)$, and streak camera sweep speed $( \pm 0.5 \%)$, with errors summed in quadrature.

Three remaining potential sources of error in $U_{s}^{\mathrm{Al}}$ - edge rarefactions, shock non-planarity and shock unsteadiness - are minimized using suitable correction procedures. Side rarefactions, which occur at the edge of the aluminum step next to the quartz sample, can perturb break-out from the top step if the measurement is taken too close to the edge. Such rarefaction waves move laterally at velocities less than the shock speed. Thus as long as break-out 
times from the top step are taken more than one step height $(35-40 \mu \mathrm{m})$ away from the edge such rarefaction effects are avoided. The effects of shock non-planarity are minimized by reducing the separation between the top and bottom step break-out time measurements, while still avoiding the rarefaction region. Non-planarity in these experiments can be described over the several hundred micron field of view by an effective shock radius of curvature of around $4 \mathrm{~mm}$ which gives a $1 \%$ error in the velocity for lateral separations of $50 \mu \mathrm{m}$. This is reduced further by tracking the spatial variation of break-out times over $\sim 100-150 \mu \mathrm{m}$ on the top and bottom steps and extrapolating them to the same point in the center of the edge region (the Distance $=0 \mu \mathrm{m}$ point in Fig. 1b). Using this approach we estimate typical non-planarity errors to be $<1 \%$.

The importance of shock steadiness effects arises from the need to compare $U_{s}^{\mathrm{Al}}$ and $U_{s}^{\mathrm{Q}}$ at similar times. More specifically, correct application of the impedance-matching condition requires that $U_{s}^{\mathrm{Al}}$ and $U_{s}^{\mathrm{Q}}$ be determined at break-out from the aluminum-quartz interface $\left(t_{\mathrm{bo}}\right)$. In Figs. 1(b) and 1(c), this is the dashed line at 4.13 ns. Determining $U_{s}^{\mathrm{Q}}\left(t_{\mathrm{bo}}\right)$ is straightforward and involves a linear extrapolation of data starting 100-200 ps after $t_{\mathrm{bo}}$; this minimizes uncertainties arising immediately after break-out where velocities are blurred both by the time resolution of the diagnostic and the presence of a 1-3 $\mu \mathrm{m}$ glue layer between the aluminum and quartz. Determining $U_{s}^{\mathrm{Al}}\left(t_{\mathrm{bo}}\right)$ requires estimating the time history, $U_{s}^{\mathrm{Al}}(t)$. This can be achieved using the measured $U_{s}^{\mathrm{Q}}(t)$ given that the same pressure source drives both materials in parallel. Since aluminum and quartz have such similar impedances, to a good approximation $U_{s}^{\mathrm{Al}}(t)-\left\langle U_{s}^{\mathrm{Al}}\right\rangle=U_{s}^{\mathrm{Q}}(t)-\left\langle U_{s}^{\mathrm{Q}}\right\rangle$ provided the average for both materials is taken over the same time period (the step transit time in this case) [31]. Although the absolute quartz velocity has a 1-2 \% error, the uncertainty in $U_{s}^{\mathrm{Q}}-\left\langle U_{s}^{\mathrm{Q}}\right\rangle$ is smaller, usually less than $1 \%$ of the velocity if the shock velocity varies smoothly. This error is summed in quadrature with the other errors in both $U_{s}^{\mathrm{Q}}$ and $U_{s}^{\mathrm{Al}}$.

The aluminum and quartz shock velocities are plotted in Fig. 2 and tabulated in Table I. The best linear fit to the data, expressed in a form where errors in the coefficients are independent, is given by $U_{s}^{\mathrm{Al}}=B_{0}+B_{1}\left(U_{s}^{\mathrm{Q}} \overline{U_{s}^{\mathrm{Q}}}\right)$ where $\overline{U_{s}^{\mathrm{Q}}}=20.57, B_{0}=21.14 \pm 0.12$ and $B_{1}=0.91 \pm 0.03$.

Having extracted $U_{s}^{\mathrm{Al}}$ and $U_{s}^{\mathrm{Q}}$ at the interface, impedance matching can now be applied to determine the quartz pressure, density, and particle speed. This requires knowledge of the aluminum Hugoniot and its release isentropes. There are two approaches to determining 
these. The first method uses a model EOS for aluminum, usually in the form of a table. This assures thermodynamic consistency in the calculations but makes it difficult to rigorously account for errors in the EOS model, a factor that becomes crucial at multi-Mbar pressures. The second method, which we use here, relies on the approximation that release curves are simply a reflection of the Hugoniot about the initial shock state in the pressure-particle velocity $\left(P-U_{p}\right)$ plane [10]. Since the Hugoniot of aluminum (and many other materials) can be described by a linear fit in $U_{s^{-}} U_{p}$ the great advantage of this approach is that the fit uncertainties give a direct way to estimate systematic errors in the aluminum EOS [33]. A possible drawback to this technique is that it does not automatically assure thermodynamic consistency between the different Hugoniot and isentrope combinations. This is unlikely to be a problem for such small variations in the Hugoniot and isentrope. The impedance match results, using a fit to absolute aluminum data given by $U_{s}=6.591+1.157 U_{p}$ [34], are shown in Table I with both the random and systematic errors given in parentheses. Random errors are a direct result of the listed velocity measurement errors; systematic errors are the result of errors in the aluminum EOS. Note how the systematic uncertainties are always smaller than the random uncertainties but rise to a third of the total error at the highest pressure near $\sim 15$ Mbar.

\section{DISCUSSION}

These data, along with results from earlier studies, are plotted in the $U_{s}-U_{p}$ plane in Fig. 3, with the best linear fit, given by $U_{s}=4.08+1.30 U_{p}$, and its associated uncertainties shown as three dashed lines [35]. The fit is in good agreement with previous data from Trunin $[15,16]$ for $13<U_{s}<20 \mu \mathrm{m} / \mathrm{ns}$ and extrapolates to the nuclear-driven point at $U_{s}$ $=33 \mu \mathrm{m} / \mathrm{ns}$. The scatter in our data is consistent with the quoted errors, giving a reduced $\chi^{2}=1.2$ (systematic errors due to the aluminum EOS, which do not contribute to data scatter, are not included in the $\chi^{2}$ calculation). Errors in the data from Trunin [16] are not provided for each shot but are quoted to be about $2 \%$ at the highest pressures, very similar to those for our measurements. These results show that to better than $2 \%$ in shock velocity, our data, achieved on timescales of less than 1 nanosecond, are in agreement with data achieved on timescales up to tens of microseconds.

By comparing data from earlier sources that have investigated compression in the solid 
phases through to the onset of melting at 1 Mbar $[9,15,16,32]$ the trend toward softening of the Hugoniot becomes apparent. It is seen most clearly by comparing the slopes of the linear $U_{s}-U_{p}$ fits in different pressure regimes (see Figs. 3 and 4). In the high-pressure solid up to melt, the data follow $U_{s}=1.370+1.822 U_{p}[9]$; in the liquid immediately above melt the best fit gives $U_{s}=2.049+1.619 U_{p}[9]$; merging into the dense plasma our data give a fit of $U_{s}=4.08+1.30 U_{p}$ over an extensive pressure range. The slope $S=\mathrm{d} U_{s} / \mathrm{d} U_{p}$ is thus decreasing with pressure. The use of piecewise linear fits is only a convenient construction and the actual Hugoniot is likely to smoothly transition to the gentler slope over a finite pressure range between 1 and $\sim 4$ Mbar. Different regimes along the quartz Hugoniot can thus be roughly characterized as: (1) Solid for $P \lesssim 1$ Mbar; (2) Liquid for $1 \lesssim P \lesssim 4$ Mbar; and (3) Dense plasma for $P \gtrsim 4$ Mbar. The softening of the Hugoniot above 1 Mbar was described theoretically by Kerley [36] who showed that without including the effects of dissociation the quartz Hugoniot continued to follow the less compressible path given approximately by the liquid fit in our plot; including dissociation caused a suppression of the temperature at a few Mbar (as a result of the dissociation energy) and a resultant softening of the Hugoniot. A recent molecular dynamics simulation has also shown that in the pressure range between 1 and 5 Mbar silica undergoes a loss of coordination between $\mathrm{Si}$ and $\mathrm{O}$ atoms [17], a process characteristic of dissociation in the dense fluid.

The data are compared to models by plotting them in the $P$ - $\rho$ plane in Fig. 4. Although the SESAME [37] model is in poor agreement with the best fit to our data, both the Kerley [36] and qEOS [38] models are in good agreement; this might be expected since the Kerley and qEOS models were guided by earlier results $[15,16]$. It is important to note that these models achieve similar $P-\rho$ behavior despite having significantly different temperature behavior. The explicit inclusion of dissociation effects by Kerley [36] causes a lowering of temperature, an effect not observed in qEOS. Note that both these models do not agree with the data in the vicinity of the melt at 1 Mbar, a disagreement possibly caused by the observed superheating of the solid phase [9].

It is instructive to examine how the Gruneisen parameter, $\Gamma$, changes along the quartz Hugoniot. In stishovite, $\Gamma$ has been determined by comparing the principal Hugoniots of quartz, coesite, and stishovite [14], and is found to decrease with volume to 0.7 near the melting point, having dropped from 1.35 at ambient pressure. At higher pressures we can approximate $\Gamma$ from the slope, $S$, of our data by the simple relation $\Gamma \simeq 2(S-1)$ [39]. 
This is rigorously true in the limit of maximum compression (when neighboring states on the Hugoniot are isochoric) but was found to be approximately valid even at considerably lower pressures such as those down to several Mbar [39]. Since $S=1.30$ between 2 and 15 Mbar it appears that, within the validity of this approximation, $\Gamma \simeq 0.6$ over this wide range. It is noteworthy that experiments comparing the Hugoniot for quartz with that for porous samples estimated $\Gamma=0.6 \pm 0.1[10,16]$ for fluid silica while available EOS models give $\Gamma \simeq 0.5-0.7[36,38]$ in this high pressure range. This indicates that $\Gamma$ drops rapidly during initial compression in the solid phase but reaches an approximately constant value at pressures just above the melt transition with dissociation at a few Mbar causing little effect [40]. It is not until pressures exceed 100 Mbar that inner-shell ionization begins to cause $\Gamma$ to decrease below 0.6 .

\section{CONCLUSIONS}

An extensive study has been performed on shocked silica in the regime where it transforms from a liquid near melt to a dense plasma. Three regimes have been identified: Solid $(P \lesssim 1$ Mbar), dissociating liquid ( $1 \lesssim P \lesssim 4$ Mbar), and dense plasma ( $P \gtrsim 4$ Mbar). The softening of the Hugoniot between 1 and 4 Mbar is likely caused by dissociation of the Si-O system. With these laser-driven shock wave measurements on quartz an accurate comparison can now be made between 3 different experimental platforms all attempting to achieve the same thermodynamic states with timescales spanning over 4 orders of magnitude. In the region between 2 and 6 Mbar, where results from all platforms exist, the data from explosivedriven, nuclear-driven, and laser-driven experiments all produce the same results to within experimental errors. Although extensive experiments have shown that quartz exhibits nonequilibrium behavior at both solid-solid [23] and solid-liquid [9] phase boundaries, once the fluid phase has been accessed the shock states achieve equilibrium in significantly less than a nanosecond.

\section{ACKNOWLEDGEMENTS}

We thank G. I. Kerley for providing his EOS table for quartz, Walter Unites for his assistance with the quartz samples, and the OMEGA target fabrication and operations 
staff for their invaluable efforts during these experiments. This work was performed under the auspices of the U.S. D.O.E. by LLNL under Contract No. W-7405-ENG-48 and by the University of Rochester under Cooperative Agreement No. DE-FC03-92SF19460.

\section{APPENDIX: DEVELOPMENT OF QUARTZ AS AN IMPEDANCE-MATCH STANDARD}

The impedance-matching technique for determining a material's Hugoniot is a relative EOS measurement whose reliability ultimately rests on the precision to which the EOS of the standard is known. Aluminum is the most often used standard since it has the largest number of absolute Hugoniot measurements [22, 41] over a wide range of pressures, particularly in the regime of one to tens of Mbar currently the focus of laser-driven [42, 43], explosively-driven [44], and magnetically-driven [45] shock techniques.

The development of the VISAR technique in laser-driven shock experiments $[27,28]$ has allowed shock speed measurements with $\sim 1 \%$ precision to be achieved and enabled continuous recording of the shock velocity with time in transparent materials. In an opaque material such as aluminum however the VISAR cannot be used and shock speeds must be obtained from the transit time across a known step height, an inherently less precise measurement (see the discussion in Section III). Since the impedance matching calculation

further magnifies errors in the shock velocity of the standard, the uncertainties in $U_{s}^{\mathrm{Al}}$ are very often the dominant component of the final errors in $\rho$ and $U_{p}$. With the extensive Hugoniot measurements obtained in this study it now becomes possible to replace aluminum with quartz to take advantage of the increased measurement precision of the VISAR. This should significantly improve the accuracy of future impedance matching measurements at least to the extent that the release or re-shock states are well-approximated by the reflected Hugoniot $[33,46]$.

For experiments with a large impedance mismatch, in which the reflected Hugoniot approximation breaks down, knowledge of the release curve (or re-shock Hugoniot) is required in addition to the principal Hugoniot determined in this study. However, experimental data on the release of quartz are thus far limited to first shock states below 1 Mbar [47]; in contrast the limit for aluminum is up to $\sim 5$ Mbar [48]. Systematic errors in the off-Hugoniot states of quartz are thus likely to be difficult to quantify, potentially off-setting the benefits 
of a more precise shock velocity measurement [49]. For such a situation we have developed a new technique that takes advantage of the increased measurement precision in the quartz sample while still relying on aluminum as the material standard. In this new scheme, a quartz 'window', identical to the sample used in these experiments, would be mounted on half a flat aluminum pusher as shown in Fig. 5, with the other half being covered by the test sample. The shock speed in aluminum immediately prior to break-out can then be determined by measuring the shock speed in quartz immediately after break-out and using the linear relation shown in Fig. 2 and provided in the text. Such a scheme has recently been used to study the principal Hugoniot of liquid deuterium.

For measurements of the double-shock behavior of liquid deuterium, quartz has already been used as an anvil [50]. In this configuration only knowledge of the principal Hugoniot is required. The precise fit for quartz was slightly different to that given in this paper since that earlier study used a preliminary set of quartz data. These differences are negligible and do not affect the results from that study.

In the schemes just described, the central principal is to develop a transparent standard for performing measurements as close as possible to the impedance-matching interface. Localizing the measurement in both space and time minimizes any effects due to spatial or temporal variations in the shock wave and eliminates the need for a transit time measurement. This approach can only grow in importance as higher pressures are accessed. 
[1] R. J. Hemley, C. T. Prewitt, K. Kingma, Rev. Mineral. 29, 41 (1994).

[2] S. Stishov and S. Popova, Geochemistry 10, 923 (1961).

[3] Q. Williams and R. Jeanloz, Science 239, 902 (1988).

[4] D. Andrault, G. Fiquet, F. Guyot, M. Hanfland, Science 282, 720 (1998).

[5] L. S. Dubrovinsky, S. K. Saxena, P. Lazor, R. Ahuja, O. Eriksson, J. M. Wills, B. Johansson, Nature, 388, 362 (1997).

[6] G. Shen and P. Lazor, J. Geophys. Res. 100, 17699 (1995).

[7] J. Wackerle, J. Appl. Phys. 33, 922 (1962).

[8] R. G. McQueen, J. N. Fritz, S. P. Marsh, J. Geophys. Res. 68, 2319 (1963).

[9] G. A. Lyzenga and T. J. Ahrens, J. Geophys. Res. 88, 2431 (1983).

[10] R. F. Trunin, Shock Compression of Condensed Materials (Camb. Univ. Press, Cambridge, 1998).

[11] D. M. Teter, R. J. Hemley, G. Kresse, J. Hafner, Phys. Rev. Lett. 80, 2145 (1998).

[12] J. A. Akins and T. J. Ahrens, Geophys. Res. Lett. 29, 31 (2002).

[13] W. R. Panero, L. R. Benedetti, R. Jeanloz, J. Geophys. Res. 108, 2015 (2003).

[14] S-N. Luo, J. L. Mosenfelder, P. D. Asimow, T. J. Ahrens, Geophys. Res. Lett. 29, 36 (2002); S-N. Luo, J. L. Mosenfelder, P. D. Asimow, T. J. Ahrens, Phys. Usp. 45, 435 (2002).

[15] R. F. Trunin, G. V. Simakov, M. A. Podurets, B. N. Moiseyev, L. V. Popov, Acad. Sci. USSR, Izvestiya, Phys. Solid Earth 1, 8 (1971).

[16] R. F. Trunin, Phys. Usp. 37, 1123 (1994).

[17] Y. Laudernet, J. Clérouin, and S. Mazevet, Phys. Rev. B 70, 165108 (2004).

[18] H. J. Melosh, Impact Cratering (Oxford University Press, Oxford, 1989).

[19] T. Lay, Q. Williams, E. J. Garnero, Nature, 392, 461 (1998).

[20] T. Lay, E. J. Garnero, Q. Williams, Phys. Earth Planet. Int. 146, 241 (2004).

[21] What we refer to as explosive- and nuclear-driven shock waves are commonly referred to as laboratory and full-scale experiments [10].

[22] M.D. Knudson, R.W. Lemke, D.B. Hayes, C.A. Hall, C. Deeney, and J.R. Asay, J. Appl. Phys. 94, 4420 (2003).

[23] G. E. Duvall and R. A. Graham, Rev. Mod. Phys. 49, 523 (1977). 
[24] Y. B. Zel'dovich and Y. P. Raizer, Physics of Shock Waves and High-Temperature Hydrodynamic Phenomena (Dover, Mineola, New York, 2002).

[25] T. R. Boehly, D. L. Brown, R. S. Craxton et al., Opt. Comm. 133, 495 (1997).

[26] L. M. Barker and R. E. Hollenbach, J. Appl Phys. 43, 4669 (1972).

[27] P. M. Celliers, G. W. Collins, L. B. Da Silva, D. M. Gold, R. Cauble, Appl. Phys. Lett. 73, 1320 (1998).

[28] P. M. Celliers, D. K. Bradley, G. W. Collins, D. G. Hicks, T. R. Boehly, W. J. Armstrong, Rev. Sci. Instrum. 75, 4916 (2004).

[29] On shots with longer time sweeps, where break-out from the rear side of the quartz could be observed, $U_{s}^{\mathrm{Q}}$ could be integrated over the transit time through the sample. This transit distance was found to be in good agreement with the known thickness of the quartz sample confirming that the VISAR-measured Doppler shift was in fact that caused by the moving shock front in quartz.

[30] Note that this time is smaller than the instrument resolution of 40-50 ps which is a product of the VISAR delay (16 or 37 ps for the etalons used in this case) and streak camera convolution and is a measure of the smallest clearly distinguishable time between two events. This is possible since the separation between break-out events is much greater than the instrument resolution and the data record is over-sampled (with $\sim 15$ pixels per resolution element in this case).

[31] Corrections for shock unsteadiness in the past have usually relied on two calculations: a hydrodynamic simulation of the experiment, and an assumed EOS for the material under study $[10,15,16]$. Our approach removes the need for a hydrodynamic simulation - since we are able to measure the adjacent shock velocity in quartz - but still relies on prior information about the EOS of quartz (which we have simply assumed to be similar to aluminum).

[32] S. P. Marsh (ed.), LASL Shock Hugoniot Data (Univ. of California Press, Berkeley, 1980).

[33] Higher order corrections to account for the slight deviation of the release isentrope from its reflected Hugoniot approximation are generally negligible unless release pressures are less than a third the original pressure at the Hugoniot [46]. Since quartz is close in impedance to aluminum this is not a concern here. For completeness these deviations were estimated by averaging the predictions from several EOS models and deriving a composite release correction curve. This small correction and its uncertainty (given by the scatter between models) were 
then added to the reflected experimental Hugoniot. Although this makes the release curve model dependent, the contributions of the models are very small. For the aluminum-quartz combination this correction is negligible. This procedure will be described in detail in a future publication by Celliers et al. and is of particular relevance to low impedance materials.

[34] The fit to the aluminum Hugoniot with associated errors was found to be $U_{s}=a_{0}+a_{1}\left[U_{p}-\overline{U_{p}}\right]$ where $a_{0}=17.409 \pm 0.081 \mu \mathrm{m} / \mathrm{ns}, a_{1}=1.157 \pm 0.031$, and $\overline{U_{p}}=9.3502 \mu \mathrm{m} / \mathrm{ns}$. This was obtained by fitting published absolute data $[22,41]$ as well as some recent unpublished points provided by M. D. Knudson (private communication).

[35] Since the errors in $U_{p}$ for the quartz Hugoniot are significantly larger than those in $U_{s}$, the fit was performed with $U_{p}$ as the dependent variable and found to be $U_{p}=C_{0}+C_{1}\left(U_{s}-\overline{U_{s}}\right)$, where $\overline{U_{s}}=20.57 \mu \mathrm{m} / \mathrm{ns}$ was the mean shock velocity for all the shots, $C_{0}=12.74 \pm 0.12$ $\mu \mathrm{m} / \mathrm{ns}$ and $C_{1}=0.772 \pm 0.027$.

[36] G. I. Kerley, Equations of State for Composite Materials (Kerley Publishing Services, Albuquerque, 1999)

[37] S. P. Lyon and J. D. Johnson, Los Alamos National Laboratory Rep. LA-UR-92-3407, Los Alamos, NM (1992).

[38] R. M. More, K. H. Warren, D. A. Young, G. B. Zimmerman, Phys. Fluids 31, 3059 (1988); D. A. Young and E. M. Corey, J. Appl. Phys 78, 3748 (1995).

[39] J. D. Johnson, Shock Compression of Condensed Materials - 1997, S. C. Schmidt, American Institute of Physics Conf. Proc. No. 429, 27 (American Institute of Physics, New York, 1998).

[40] The model by Kerley [36] predicts that $\Gamma \simeq 0.5$ during dissociation between 1 - 5 Mbar, rising to 0.75 then declining at higher pressures to between 0.6-0.7.

[41] V. A. Simonenko, N. P. Voloshin, A. S. Vladimirov, A. P. Nagibin, V. N. Nogin, V. A. Popov, V. A. Vasilenko, Yu. A. Shoidin, Sov. Phys. JETP 61, 869 (1985); M. A. Podurets, V. M. Ktitorov, R. F. Trunin, L. V. Popov, A. Ya. Matveev, B. V. Pechenkin, A. G. Sevast'yanov, High Temperature 32, 890 (1994).

[42] D. G. Hicks, P. M. Celliers, G. W. Collins, J. H. Eggert, S. J. Moon, Phys. Rev. Lett. 91, $035502(2003)$.

[43] P. M. Celliers, G. W. Collins, D. G. Hicks et al., Phys. Plasmas 11, L41 (2004).

[44] S. I. Belov, G. V. Boriskov, A. I. Bykov et al., JETP Lett. 76, 433 (2002); G. V. Boriskov, A. I. Bykov, R. I. Il'kaev et al. Doklady Phys. 48, 553 (2003). 
[45] M. D. Knudson, D. L. Hanson, J. E. Bailey, C. A. Hall, J. R. Asay, Phys. Rev. Lett. 87, 225501 (2001); M. D. Knudson, D. L. Hanson, J. E. Bailey, C. A. Hall, J. R. Asay, Phys. Rev. Lett. 90, 035505 (2003).

[46] M. V. Zhernokletov, G. V. Simakov, Y. N. Sutulov, R. F. Trunin, High Temp. 33, 36 (1995); V. A. Bugaeva, A. A. Evstigneev, and R. F. Trunin, High Temp. 34, 674 (1995).

[47] M. A. Podurets, G. V. Simakov, R. F. Trunin, Izv. Earth Phys. 12, 419 (1976).

[48] M. D. Knudson, J. R. Asay, C. Deeney, J. Appl. Phys. 97, 073514 (2005)

[49] Although there are no release data for quartz above $1 \mathrm{Mbar}$, there exist extensive Hugoniot measurements on porous samples in the Mbar range [10]. Using an EOS model, these data can be used to constrain release paths for quartz. By combining predicitions from different models, uncertainties in the release curves can be estimated.

[50] T. R. Boehly, D. G. Hicks, P. M. Celliers, T. J. B. Collins, R. Earley, J. H. Eggert, D. JacobsPerkins, S. J. Moon, E. Vianello, D. D. Meyerhofer, G. W. Collins, Phys. Plasmas 11, L49 (2004).

This work was performed under the auspices of the U. S. Department of Energy by University of California, Lawrence Livermore National Laboratory under contract W-7405-Eng-48. 
TABLE I: Summary of experimental shock wave data on quartz. $U_{s}^{\mathrm{Al}}$ and $U_{s}^{\mathrm{Q}}$ are the primary experimental observables; measurement uncertainties are listed in parentheses. $P^{\mathrm{Q}}, \rho^{\mathrm{Q}}$, and $U_{p}^{\mathrm{Q}}$ are inferred from impedance matching with aluminum; random and systematic errors for these quantities are listed in parentheses first and second respectively. The random errors arise from the listed measurement uncertainties in the primary observables. Systematic errors arise from uncertainties in the experimentally-derived aluminum Hugoniot.

\begin{tabular}{cccccc}
\hline \hline Shot & $U_{s}^{\mathrm{Al}}$ & $U_{s}^{\mathrm{Q}}$ & $P^{\mathrm{Q}}$ & $\rho^{\mathrm{Q}}$ & $U_{p}^{\mathrm{Q}}$ \\
$\#$ & $(\mu \mathrm{m} / \mathrm{ns})$ & $(\mu \mathrm{m} / \mathrm{ns})$ & $(\mathrm{Mbar})$ & $(\mathrm{g} / \mathrm{cc})$ & $(\mu \mathrm{m} / \mathrm{ns})$ \\
\hline 31913 & $14.33(0.34)$ & $12.62(0.32)$ & $2.37(0.12,0.02)$ & $6.05(0.44,0.06)$ & $7.09(0.33,0.05)$ \\
31917 & $14.88(0.30)$ & $13.50(0.26)$ & $2.68(0.12,0.04)$ & $5.96(0.38,0.10)$ & $7.49(0.33,0.10)$ \\
29011 & $15.59(0.35)$ & $14.59(0.32)$ & $3.11(0.15,0.03)$ & $5.90(0.40,0.07)$ & $8.04(0.38,0.08)$ \\
29031 & $15.94(0.43)$ & $14.90(0.41)$ & $3.30(0.19,0.03)$ & $6.03(0.51,0.07)$ & $8.35(0.47,0.08)$ \\
27890 & $17.76(0.35)$ & $17.56(0.26)$ & $4.54(0.18,0.03)$ & $5.97(0.32,0.06)$ & $9.77(0.38,0.07)$ \\
29411 & $17.82(0.29)$ & $17.52(0.24)$ & $4.57(0.15,0.03)$ & $6.05(0.29,0.06)$ & $9.84(0.32,0.07)$ \\
29400 & $18.11(0.32)$ & $17.96(0.26)$ & $4.79(0.17,0.04)$ & $6.03(0.31,0.06)$ & $10.07(0.35,0.07)$ \\
29025 & $18.08(0.29)$ & $17.52(0.25)$ & $4.70(0.15,0.03)$ & $6.28(0.32,0.06)$ & $10.13(0.32,0.07)$ \\
31911 & $18.37(0.29)$ & $17.13(0.23)$ & $4.77(0.15,0.04)$ & $6.87(0.39,0.08)$ & $10.52(0.32,0.08)$ \\
27898 & $19.03(0.34)$ & $18.25(0.24)$ & $5.32(0.19,0.04)$ & $6.67(0.39,0.08)$ & $11.00(0.38,0.08)$ \\
29014 & $20.22(0.34)$ & $19.46(0.27)$ & $6.20(0.20,0.05)$ & $6.94(0.40,0.09)$ & $12.03(0.37,0.10)$ \\
29418 & $21.13(0.37)$ & $20.34(0.26)$ & $6.92(0.23,0.06)$ & $7.19(0.44,0.11)$ & $12.84(0.41,0.11)$ \\
29029 & $22.27(0.33)$ & $22.02(0.26)$ & $8.00(0.22,0.08)$ & $7.03(0.35,0.11)$ & $13.71(0.36,0.13)$ \\
27900 & $22.56(0.43)$ & $22.76(0.26)$ & $8.36(0.29,0.08)$ & $6.78(0.39,0.11)$ & $13.86(0.47,0.14)$ \\
$2941923.05(0.40)$ & $23.12(0.24)$ & $8.78(0.28,0.09)$ & $6.97(0.38,0.12)$ & $14.33(0.44,0.15)$ \\
29022 & $23.72(0.33)$ & $22.74(0.27)$ & $9.12(0.23,0.10)$ & $7.93(0.45,0.17)$ & $15.14(0.37,0.16)$ \\
29413 & $23.90(0.42)$ & $23.03(0.24)$ & $9.32(0.29,0.10)$ & $7.86(0.51,0.17)$ & $15.27(0.46,0.17)$ \\
27896 & $24.27(0.45)$ & $23.84(0.23)$ & $9.79(0.31,0.11)$ & $7.57(0.48,0.16)$ & $15.50(0.49,0.17)$ \\
29008 & $25.27(0.45)$ & $25.61(0.25)$ & $10.99(0.34,0.13)$ & $7.21(0.41,0.15)$ & $16.20(0.49,0.19)$ \\
27894 & $25.96(0.61)$ & $25.54(0.23)$ & $11.48(0.46,0.14)$ & $7.89(0.64,0.19)$ & $16.96(0.67,0.21)$ \\
29420 & $26.16(0.74)$ & $26.01(0.25)$ & $11.77(0.56,0.15)$ & $7.71(0.72,0.18)$ & $17.08(0.81,0.21)$ \\
\hline \hline & $28.49(0.49)$ & $28.26(0.26)$ & $14.32(0.42,0.20)$ & $8.19(0.53,0.23)$ & $19.12(0.54,0.27)$ \\
$2250.80(0.48)$ & $28.92(0.26)$ & $14.81(0.41,0.21)$ & $7.98(0.48,0.23)$ & $19.32(0.53,0.27)$ \\
\hline
\end{tabular}




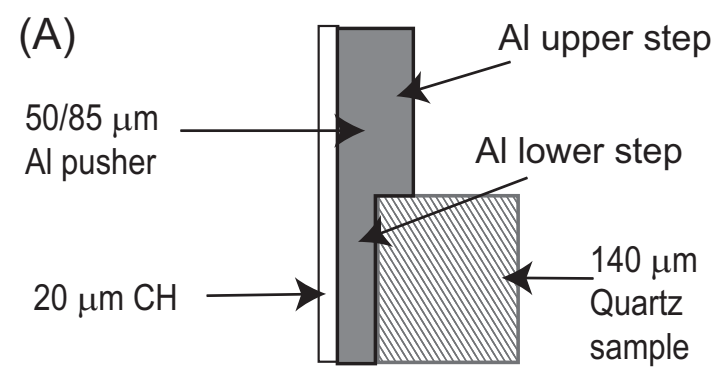

(B)

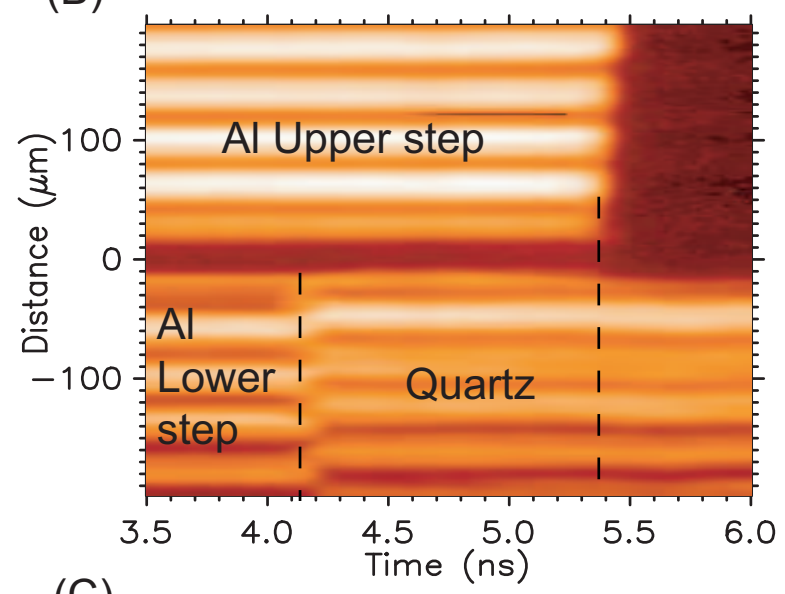

(C)

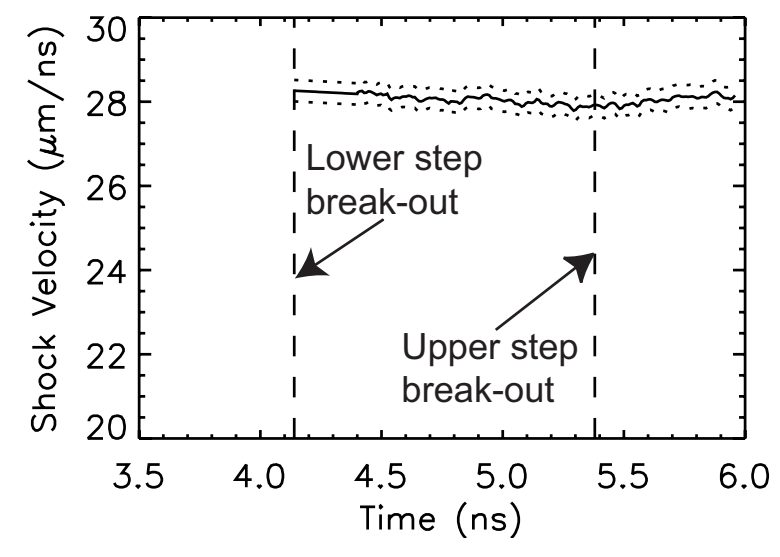




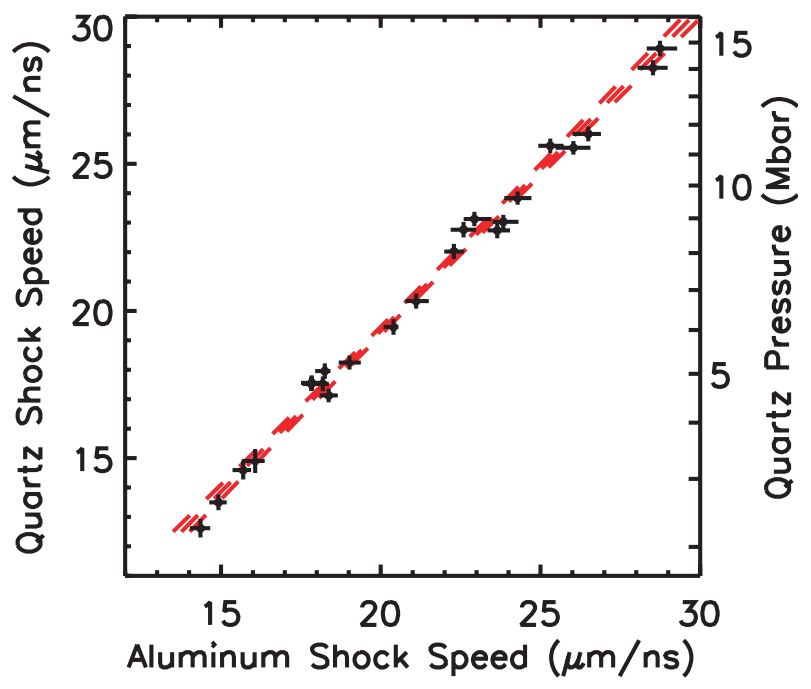




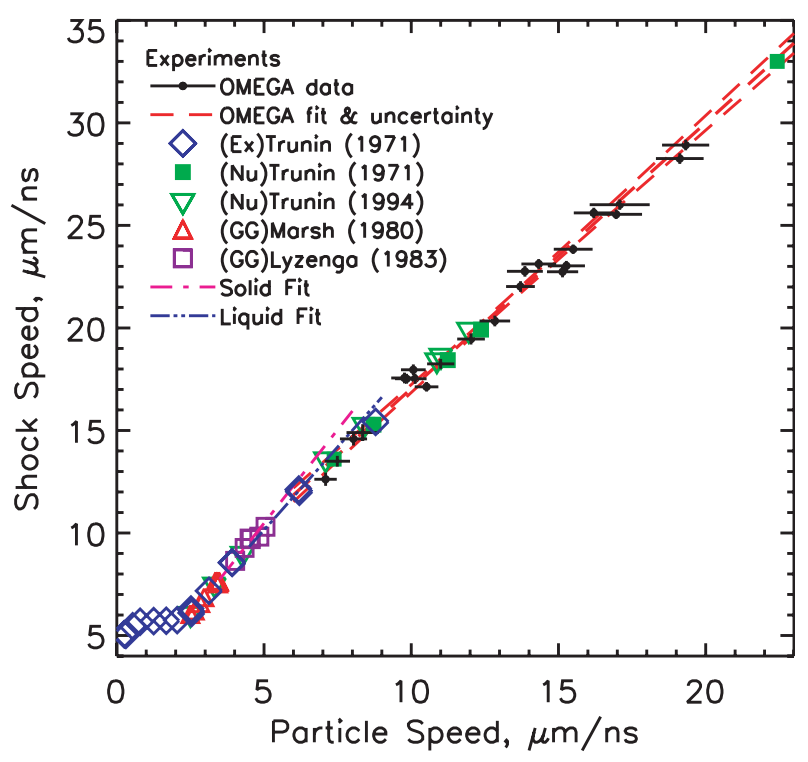




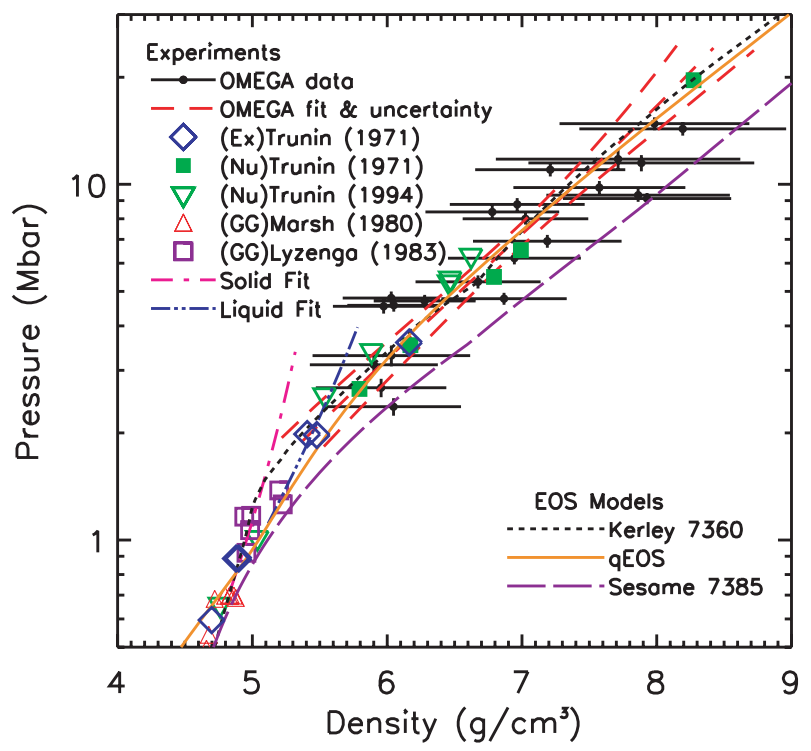




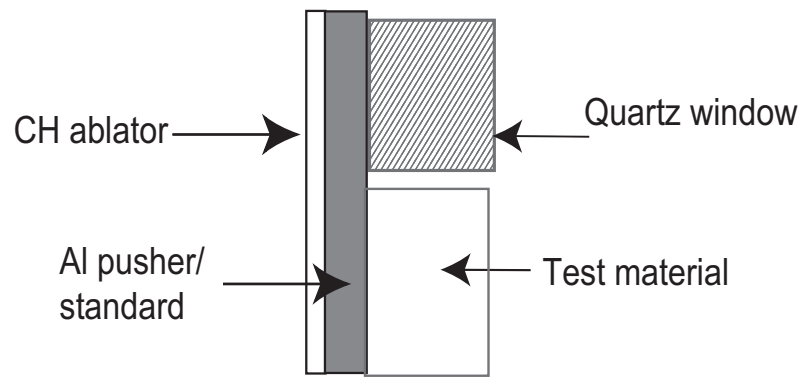


FIG. 1: (A) Schematic of target: Drive laser is incident from the left; VISAR views from the right. (B) Streak camera record of the line-VISAR trace showing break-out from upper and lower aluminum steps and the reflecting shock wave in quartz. (C) Shock velocity in quartz inferred from the VISAR fringe shift in (B) with uncertainties given by the dotted lines.

FIG. 2: Plot of the experimental observables determined at the aluminum-quartz interface: Shock Velocity in the aluminum standard, as determined by the transit time across the aluminum step, versus shock velocity in quartz as measured by the VISAR. The data can be represented by a straight line with fit parameters given in the text.

FIG. 3: Measured quartz Hugoniot shown in the shock velocity versus particle velocity plane. The red dashed lines give the best linear fit and associated $1 \sigma$ uncertainty to the data from this study (black circles with error bars). Previous data from Trunin $[15,16]$ performed on explosively-driven (Ex) and nuclear-driven $(\mathrm{Nu})$ platforms are in good agreement with those from this study to within experimental uncertainties. Selected data at lower pressures obtained on gas gun experiments (GG) are also shown from Lyzenga [9] and Marsh [32]. Linear fits determined by Lyzenga [9] are shown for the solid (stishovite or stishovite-like) phase (pink, dot dash line) and the liquid phase (dark blue, double-dot dash line) immediately after melting.

FIG. 4: Measured quartz Hugoniot from Fig. 3 in the pressure-density plane illustrating the softening of the quartz Hugoniot as it proceeds through melt to the liquid and dense plasma phases. Predictions of some EOS models are shown for comparison. As in Fig. 3, previous data from explosive (Ex), nuclear (Nu), and gas gun (GG) experiments are shown and are in good agreement with the laser-driven data from this study.

FIG. 5: A new, high-precision scheme for determining aluminum shock velocities in an impedancematch experiment. In this arrangement, the VISAR-measured shock velocity in the quartz window is used to infer the shock velocity in aluminum prior to break-out, the relationship between aluminum and quartz shock velocities being given by the statistically-averaged fit shown in Fig. 2 and provided in the text. This significantly reduces the error arising from transit time measurements across a step. 